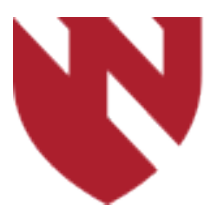

December 2019

\title{
Renal Salvage after Stent Graft Placement after Acute Renal Artery Occlusion with Prolonged Time of Ischemia
}

\author{
Yue Gao \\ University of Nebraska Medical Center \\ Dimitrios Miserlis \\ University of Nebraska Medical Center \\ Matthew Longo \\ University of Nebraska Medical Center \\ Nitin Garg \\ University of Nebraska Medical Center
}

Tell us how you used this information in this short survey.

Follow this and additional works at: https://digitalcommons.unmc.edu/gmerj

Part of the Higher Education Commons, and the Medicine and Health Sciences Commons

\section{Recommended Citation}

Gao, Y., Miserlis, D., Longo, M., , Garg, N. Renal Salvage after Stent Graft Placement after Acute Renal Artery Occlusion with Prolonged Time of Ischemia. Graduate Medical Education Research Journal. 2019 Dec 13; 1(1).

https://digitalcommons.unmc.edu/gmerj/vol1/iss1/41

This Conference Proceeding is brought to you for free and open access by DigitalCommons@UNMC. It has been accepted for inclusion in Graduate Medical Education Research Journal by an authorized editor of DigitalCommons@UNMC.For more information, please contact digitalcommons@unmc.edu. 
Renal Salvage after Stent Graft Placement after Acute Renal Artery Occlusion with Prolonged Time of Ischemia

\section{Creative Commons License}

\section{(c) (1) $\Theta($}

This work is licensed under a Creative Commons Attribution-Noncommercial-No Derivative Works 4.0 License. 
be collected at clinic visits up to 90 days postoperatively. Data from each stage will be compared to assess the effectiveness of the guidelines in providing adequate analgesia while minimizing opioid prescriptions.

Results \& Conclusions: Pending further data collection.

https://doi.org/10.32873/unmc.dc.gmerj.1.1.038

\section{An Immediate Weight Bearing Protocol versus Stress Radiography to Determine Ankle Stability in Patients with Isolated SER Distal Fibula Fractures \\ Phillip Thomas, Leonid Grossman, Justin Siebler, Ivan Tarkin, Lori Reed, Liz Lyden, Matthew Mormino}

Mentor: Matthew Mormino

Program: Orthopaedic Surgery

Purpose: To compare stress radiographs with weight bearing radiographs in the acutely injured ankle and determine if stress views may be unnecessary and ultimately lead to unnecessary surgical intervention.

To determine if patients allowed to weight bear will show similar results radiographically and clinically to the current gold standard stress views.

Methods: A prospective study will be conducted with UNMC, UPMC \& Mississippi
Centers with 200 total subjects enrolled. Every subject with isolated fibular fracture will have stress view $\mathrm{x}$-rays taken in the ED. They will then be placed in a CAM boot and follow up in clinic $\sim 7$ days later. Weight bearing x-rays will be obtained at follow up and UNMC \& UPMC will use weight bearing $\mathrm{X}$-rays to determine operative intervention. Mississippi will use only stress views to determine operative intervention

Results \& Conclusions: Collecting data. Currently nine subjects had medial clear space widening $>4 \mathrm{~mm}$ on gold standard stress views. However, $0 / 9$ at most recent follow up have had MCS $>4 \mathrm{~mm}$ and therefore none have shown signs indicative of a need for surgery.

Hypothesis: The gold standard of stress views for isolated fibular fractures may be misrepresenting the common biomechanical stressors carried through the ankle as part of activities of daily living. Therefore, we believe it is possible that many isolated fibular fractures are being unnecessarily treated with operative intervention.

https://doi.org/10.32873/unmc.dc.gmerj.1.1.039

\section{Effect of Crystalloid Infusion Rate in a Noncompressible Hemorrhage Model} Ujwal Yanala, Jason Johanning, Iraklis Pipinos, Gustavo Larsen, William Velander, Mark Carlson

\section{Mentor: Mark Carlson \\ Program: General Surgery}

Purpose: To determine the effect of fluid administration rate on survival, vital signs, blood loss, and laboratory parameters in a porcine model of noncompressible hemorrhage.

Methods: Twenty domestic swine (barrow, age 3 months, 32-36 kg) underwent hemitransection of left lateral liver lobe without treatment. Incision was temporarily closed immediately after injury. At 60's after injury, lactated ringers solution was begun at either 150 or $20 \mathrm{~mL} / \mathrm{min}$ IV (rapid and slow group, respectively, $\mathrm{N}=10$ each); maximum volume was capped at $100 \mathrm{~mL} / \mathrm{kg}$. Both groups were monitored for 60 or $180 \mathrm{~min}$.

Results: Pre-injury physiological parameters did not differ between the groups. Survival after one hour in both the groups was $60 \%$; no further death occurred in the slow group with observation out to $180 \mathrm{~min}$. Necropsy demonstrated that an equivalent number of portal vein and hepatic vein branches had been transected in each group. There were no significant differences between groups for heart rate, temperature, total volume of LR infused, or liver weight.

Conclusion: Although the two groups were not directly comparable (no formal randomization; longer observation time in the slow group), this study demonstrated that in a porcine model of noncompressible truncal hemorrhage, intravenous crystalloid resuscitation with a relatively slow infusion rate $(20 \mathrm{~mL} / \mathrm{min})$ produced less blood loss and an improved laboratory profile (hemoglobin and protime). This study supports the U.S. military's recent adoption of a "hypotensive resuscitation" protocol for warfighters injured in the field, which dictates that fluid resuscitation of an injured warfighter with hemorrhagic shock should be restricted until the subject arrives at a forward surgical unit.

https://doi.org/10.32873/unmc.dc.gmerj.1.1.040

\section{Renal Salvage after Stent Graft Placement after Acute Renal Artery Occlusion with Prolonged Time of Ischemia}

Yue Gao, Dimitrios Miserlis, Matthew Longo, Nitin Garg

Mentor: Nitin Garg

Program: General Surgery

Purpose: To describe a patient with acute renal artery occlusion (RAO) who underwent successful revascularization procedure, after experiencing prolonged ischemic period, which resulted in successful retrieval of renal function.
Case Report: A middle-aged patient with past history left renal artery stenosis and stent graft placement presented with symptoms of chest pain, shortness of breath, and flank pain. The patient was admitted to Intensive Care Unit with diagnosis of multi-organ failure and subsequent anuria that led to initiation of hemodialysis. Computed tomography angiography (CTA) demonstrated chronic aortic occlusion along with bilateral proximal renal artery occlusion with reconstitution of the mid to distal renal arteries via collateralization. Patient underwent angioplasty with bilateral renal artery stent-graft placement and successful revascularization of proximal renal arteries. Post-operatively, renal function and urine output improved, and the patient was able 
to be weaned off hemodialysis along with the benefit of concurrent amelioration of renovascular hypertension.

Conclusion: For select patients with RAO there can benefits, such as achieving dialysis independence and stabilization of renovascular hypertension, by attaining revascularization of the renal arteries despite prolonged time of ischemia.

https://doi.org/10.32873/unmc.dc.gmerj.1.1.041

No Survival Advantage Exists for Patients Undergoing Loop lleostomy for Clostridium difficile Colitis Bradley R. Hall, Andrew Cannon, Pranita Atri, Christopher S. Wichman, Lynette M. Smith, Apar K. Ganti, Chandrakanth Are, Aaron R. Sasson, Sushil Kumar, Surinder K. Batra

\section{Mentor: Dmitry Oleynikov \\ Program: General Surgery}

Background \& Objectives: Loop ileostomy (LI) remains an unproved alternative to total abdominal colectomy (TAC) for patients with Clostridium difficile infection (CDI). We aim to compare outcomes between loop ileostomy and total abdominal colectomy for Clostridium difficile infection using a national database.

Methods: The 2011-2016 ACS-NSQIP database was queried for patients who underwent LI or TAC for CDI. Patients with high outlying age, LOS, and operative time were excluded. Statistics were performed using IBM SPSS and NCSS PASS 11.

Results: Of 457 patients identified, 47 underwent LI. Predicted morbidity was higher in the TAC cohort $(62 \%$ vs. $37 \%, \mathrm{p}<0.001)$. Patients in the LI cohort experienced fewer complications $(72 \%$ vs. $87 \%, p=0.021)$; however, mortality did not differ between LI (36\%) and TAC (31\%) cohorts. Blood transfusions were more than twice as frequent in the TAC cohort (54\% vs. 19\%, $\mathrm{p}<0.001)$. Four patients in the LI cohort required reoperation; however, none required colectomy.

Conclusions: Compared to TAC, LI does not improve survival for patients who require surgical intervention for CDI.

https://doi.org/10.32873/unmc.dc.gmerj.1.1.042

\section{Advanced Pancreatic Cancer: A Meta-Analysis of Clinical Trials over Thirty Years Bradley R. Hall, Jennifer A. Leinicke, Priscila R. Armijo, Lynette M Smith, Sean J. Langenfeld, Dmitry Oleynikov}

Mentor: Surinder Batra

Program: General Surgery

Background \& Objectives: In contrast to other cancers, survival rates for pancreatic ductal adenocarcinoma (PDAC) have improved but minimally over the past thirty years. The aim of this study was to perform a meta-analysis of clinical trials published since 1986 to determine trends in median overall survival in primarily metastatic PDAC.

Methods: All Phase 2-4 clinical trials published during or after 1986 investigating first-line systemic chemotherapy in metastatic PDAC were included in the meta-analysis. Publications obtained through PubMed and
www.ClinicalTrials.gov were cross-referenced to identify additional trials. Trials enrolling fewer than $50 \%$ of study participants with metastatic disease were excluded.

Results: Of 19,488 patients enrolled in 151 clinical trials, $84 \%$ had metastatic disease and $16 \%$ had locally advanced pancreatic cancer. In clinical trials published from 1986 to 2016 , the weighted median overall survival (wMOS) increased by 3.0 months. The median wMOS was higher in combination therapy (7.31 months, IQR 5.4 to 8.5 ) compared to non-gemcitabine, single-agent therapy (4.76 months, IQR 3.5 to 6.0 ), gemcitabine monotherapy (6.48 months, IQR
5.9 to 7.2 ), and gemcitabine plus single-agent therapy (7.09 months, IQR 6.3 to 8.2). Of all regimens used in more than one study arm, FOLFIRINOX had the highest wMOS (10.9 months).

Conclusions: Regardless of treatment regimen, survival rates in PDAC have minimally improved over time. Of drugs used in two or more study arms, only FOLFIRINOX has a wMOS greater than ten months. Emphasis should, therefore, be placed on identification of novel targets that promote early diagnosis and intervention.

https://doi.org/10.32873/unmc.dc.gmerj.1.1.043

\section{National Trends in Survival for Pancreatic Adenocarcinoma Based on Location of the Primary Lesion} Gautam K. Malhotra, Lynette M. Smith, Quan P. Ly, Aaron R. Sasson, Chandrakanth Are

Mentor: Chandrakanth Are

Program: General Surgery

Background: The management and prognosis of pancreatic adenocarcinoma varies depending on the location of the primary lesion within the pancreas. There is a paucity of data on survival trends in pancreatic adenocarcinoma based on the location. The aim of this study was to analyze the national trends in survival for patients with pancreatic adenocarcinoma based on the location of the primary lesion.

Methods: The Surveillance, Epidemiology, and End Results (SEER) database (SEER 17, 1973-2007) was used to identify patients with pancreatic cancer located in the head (C25.0), body (C25.1), or tail (C25.2). SEER*Stat
6.6.2 was used to calculate 5-year survival data. To stabilize rates, we used 5-year cohorts when calculating trends.

Results: A total of 89,733 patients were included in the study. The overall 5-year survival, inclusive of all locations and treatment approaches was is $5.4 \%(95 \% \mathrm{CI}$ $5.2-5.6 \%)$. For lesions in the head, body and tail the overall 5-year survival, inclusive of 